\title{
REPRODUCTIVITY FOR A NEMATIC LIQUID CRYSTAL MODEL
}

\author{
Blanca Climent-EzQuerra*, \\ Francisco GuILlÉN-GONZÁLEZ* \\ MARKO ROJAS-MEDAR ${ }^{\dagger}$
}

\begin{abstract}
In this paper we prove existence of weak solution with the reproductivity in time property, for a penalized PDE's system related to a nematic liquid crystal model.

This problem is relatively explicit when time-independent Dirichlet boundary conditions are imposed for the orientation of crystal molecules. Nevertheless, for the time-dependent case, the treatment of the problem is completely different. The verification of a maximum principle for weak reproductive solutions is fundamental in the argument.

Finally, the relation between reproductive and periodic in time (regular) solutions will be pointed out, differenting the $2 D$ and $3 D$ cases. Basically, in two-dimensional domains every reproductive solution is regular and time periodic, whereas the problem remains open for three-dimensional domains.
\end{abstract}

Mathematical Subject Classification (2000): Primary 35Q35, Secondary 76D03, $35 \mathrm{~K} 20,35 \mathrm{~A} 35$

Keywords: time reproductive solutions, Navier-Stokes equations, Liquid crystal model, coupled non-linear system, Galerkin method.

*Dpto. Ecuaciones Diferenciales y Análisis Numérico, Universidad de Sevilla, Aptdo. 1160, 41080 Sevilla, Spain. E-mails: blanca@us.es, guillen@us.es

${ }^{\dagger}$ Dpto. Matemática Aplicada, IMECC-UNICAMP, C.P. 6065, 13081-970, Campinas-SP, Brazil. E-mail: marko@ime.unicamp.br 


\section{Introduction}

In this work, a nematic liquid crystal model in a simplified Ericksen-Leslie version is considered; see for instance [9] for a formulation of a more complete liquid crystal problem.

This model can be seen as a variant of the Navier-Stokes problem (respect to the unknowns velocity-pressure $(\boldsymbol{u}, p)$ ) coupled with a convection-diffusion system for a new variable $\boldsymbol{d}$, a unit vectorial function modelling the orientation of the crystal molecules. On the other hand, it is usual to consider an approximation by Ginzburg-Landau penalization ([1]) for the constraint $|\boldsymbol{d}|=1(|\boldsymbol{d}|=|\boldsymbol{d}(t, x)|$ denotes the point-wise euclidean norm).

This penalized model (in which the constraint $|\boldsymbol{d}|=1$ is relaxed by $|\boldsymbol{d}| \leq 1$ ) was introduced by Lin in [7] and studied (from a mathematical point of view) by Lin and Liu in [8,9] and by Coutand and Shkoller in [2]. The main difficulties of the model coming from the strongly nonlinear coupling between the orientation vector $\boldsymbol{d}$ and the velocity-pressure $(\boldsymbol{u}, p)$ and from the constraint $|\boldsymbol{d}| \leq 1$, jointly with the well known difficulties for the Navier-Stokes problem (a nonlinear parabollic system with the free divergence constraint related to the pressure).

In all previous works, initial conditions and time-independent Dirichlet boundary conditions for $\boldsymbol{d}$ are considered. Now, we are interested in time reproductive solutions. In particular, we will see that this study is completely different for timeindependent or time-dependent boundary conditions for $\boldsymbol{d}$.

We assume a (newtonian) fluid confined in an open bounded domain $\Omega \subset \mathbb{R}^{N}$ $(N=2$ or 3$)$ with regular boundary $\partial \Omega$. In the penalized model the constraint $|\boldsymbol{d}|=1$ is partially conserved to $|\boldsymbol{d}| \leq 1$ as consequence of the maximum principle for the Ginzburg-Landau equation considering the penalization function

$$
f(\boldsymbol{d})=\frac{1}{\varepsilon^{2}}\left(|\boldsymbol{d}|^{2}-1\right) \boldsymbol{d}
$$

where $\varepsilon>0$ is the penalization parameter. There exists a potential function

$$
F(\boldsymbol{d})=\frac{1}{4 \varepsilon^{2}}\left(|\boldsymbol{d}|^{2}-1\right)^{2}
$$

such that $f(\boldsymbol{d})=\nabla_{\boldsymbol{d}}(F(\boldsymbol{d}))$ for each $\boldsymbol{d} \in \mathbb{R}^{N}$. Then, we consider the following PDE system in $(0, T) \times \Omega$ :

$$
\left\{\begin{array}{l}
\partial_{t} \boldsymbol{u}+(\boldsymbol{u} \cdot \nabla) \boldsymbol{u}-\nu \Delta \boldsymbol{u}+\nabla p=-\lambda \nabla \cdot(\nabla \boldsymbol{d} \odot \nabla \boldsymbol{d}), \quad \nabla \cdot \boldsymbol{u}=0, \\
\partial_{t} \boldsymbol{d}+(\boldsymbol{u} \cdot \nabla) \boldsymbol{d}=\gamma(\Delta \boldsymbol{d}-f(\boldsymbol{d})), \quad|\boldsymbol{d}| \leq 1,
\end{array}\right.
$$

The constants $\nu, \lambda$ and $\gamma$ are positives, representing respectively, the fluid viscosity, an elasticity constant and a relaxation time. Here, the following tensorial notation 
is used:

$$
(\nabla \boldsymbol{d} \odot \nabla \boldsymbol{d})_{i j}=\sum_{k=1}^{N} \partial_{x_{i}} d_{k} \partial_{x_{j}} d_{k}, \quad \forall i, j=1, \ldots, N .
$$

The problem (1) is completed with the (Dirichlet) boundary conditions

$$
\boldsymbol{u}(x, t)=0, \quad \boldsymbol{d}(x, t)=\boldsymbol{h}(x, t) \quad \text { on } \partial \Omega \times(0, T)
$$

(assuming as novelty a time-depending boundary data for $\boldsymbol{d}$ given by $\mathbf{h}: \partial \Omega \times$ $(0, T) \mapsto \mathbb{R}^{N}$, in $[8,9]$ only a time-independent boundary data is considered) and the reproductivity conditions:

$$
\boldsymbol{u}(x, 0)=\boldsymbol{u}(x, T), \quad \boldsymbol{d}(x, 0)=\boldsymbol{d}(x, T) \quad \text { in } \Omega
$$

These reproductive conditions jointly with the constraint $|\boldsymbol{d}| \leq 1$ are the main difficulties of the problem (1)-(3), while the time-dependent boundary conditions $\boldsymbol{d}=\boldsymbol{h}(x, t)$ produces some additional difficulties. In particular, an adequate lifting of this condition must be done.

It is important to remark that reproductive solution with the following boundary data independent of time $\boldsymbol{d}(x, t)_{\mid \partial \Omega \times(0, T)}=\boldsymbol{d}_{0}(x)$ has the trivial stationary (static) solution:

$$
\begin{aligned}
& \boldsymbol{u} \equiv 0, \\
& \boldsymbol{d} \quad \text { solution of the elliptic problem: }-\Delta \boldsymbol{d}+f(\boldsymbol{d})=0 \quad \text { in } \Omega, \quad \boldsymbol{d}_{\mid \partial \Omega}=\boldsymbol{d}_{0}, \\
& p \quad \text { such that } \nabla p=-\lambda \nabla \cdot(\nabla \boldsymbol{d} \odot \nabla \boldsymbol{d}) .
\end{aligned}
$$

Therefore, in this work will be fundamental assume time-dependent boundary data for $\boldsymbol{d}$.

The concept of reproductive solution in the Navier-Stokes context, appears for the first time in [5], see also [10].

The goal of this paper is to obtain existence of (global in time) weak solution of problem (1)-(3). We start defining a variational formulation, testing the maximum principle for $\boldsymbol{d}$ and the energy inequality of the system. Afterwards, we introduce a Galerkin discretization of the problem, proving existence and uniqueness of approximate solution associated to arbitrary initial conditions. Then, a Leray-Schauder argument (by means of fixed point process about initial and final in time values of the solutions) allows us to obtain a reproductive Galerkin solution, which converges towards a continuous reproductive solution. Finally, some comments about the relation between reproductive and periodic (regular) solutions will be pointed out, being completely different the $2 D$ and $3 D$ cases. Basically, in two-dimensional domains every reproductive solution associated to regular boundary data is regular and time periodic, whereas the problem remains open for three-dimensional domains. 
In our opinion, other interesting open problem related with this work, is the asymptotic behavior of reproductive solutions of (1)-(3) when $\varepsilon \rightarrow 0$. In the case of initial-boundary problem, existence of weak solution of the limit problem is obtained in [3], passing to the limit when $\varepsilon \rightarrow 0$.

\section{Variational Formulation}

For simplicity, we denote $\boldsymbol{L}^{2}$ and $\boldsymbol{H}^{1}$ instead of $L^{2}(\Omega)^{N}$ and $H^{1}(\Omega)^{N}, L^{\infty}\left(\boldsymbol{H}^{1}\right)$ instead of $L^{\infty}\left(0, T ; H^{1}(\Omega)^{N}\right)$, etc. Also, the scalar product in $L^{2}$ will be denoted by $(\cdot, \cdot)$, and $\langle\cdot, \cdot\rangle$ will denote some duality products.

Let us consider the following function spaces:

$$
\begin{aligned}
\boldsymbol{H} & =\left\{\boldsymbol{u} \in \boldsymbol{L}^{2}: \nabla \cdot \boldsymbol{u}=0 \text { in } \Omega, \boldsymbol{u} \cdot \mathbf{n}=0 \text { on } \partial \Omega\right\} \\
\boldsymbol{V} & =\left\{\boldsymbol{u} \in \boldsymbol{H}_{0}^{1}: \nabla \cdot \boldsymbol{u}=0 \text { in } \Omega\right\}
\end{aligned}
$$

Without less of generality, we fix the constants $\nu=\lambda=\gamma=1$.

Obviously, the following compatibility conditions will be imposed in this work for the boundary data function $\boldsymbol{h}$ :

$$
|\boldsymbol{h}| \leq 1 \quad \text { on } \partial \Omega \times(0, T) \quad \text { and } \quad \boldsymbol{h}(0)=\boldsymbol{h}(T) \quad \text { on } \partial \Omega .
$$

\subsection{The variational problem for $(u, d)$}

Definition 1 We say that $(\boldsymbol{u}, \boldsymbol{d})$ is a weak reproductive solution of (1)-(3) if

$$
\begin{aligned}
& \boldsymbol{u} \in L^{2}(\boldsymbol{V}) \cap L^{\infty}(\boldsymbol{H}) \\
& \boldsymbol{d} \in L^{\infty}\left(H^{1}\right), \quad \Delta \boldsymbol{d} \in L^{2}\left(L^{2}\right),\left.\quad \boldsymbol{d}\right|_{\partial \Omega \times(0, T)}=\boldsymbol{h}
\end{aligned}
$$

verifying

$$
\begin{aligned}
& \left\langle\partial_{t} \boldsymbol{u}, \boldsymbol{v}\right\rangle+((\boldsymbol{u} \cdot \nabla) \boldsymbol{u}, \boldsymbol{v})+(\nabla \boldsymbol{u}, \nabla \boldsymbol{v})+\left(\nabla \boldsymbol{d}^{t} \Delta \boldsymbol{d}, \boldsymbol{v}\right)=0 \quad \forall \boldsymbol{v} \in \boldsymbol{V} \cap \boldsymbol{L}^{\infty} \\
& \left\langle\partial_{t} \boldsymbol{d}, \boldsymbol{e}\right\rangle+((\boldsymbol{u} \cdot \nabla) \boldsymbol{d}, \boldsymbol{e})+(f(\boldsymbol{d}), \boldsymbol{e})-(\Delta \boldsymbol{d}, \boldsymbol{e})=0 \quad \forall \boldsymbol{e} \in \boldsymbol{L}^{3} \\
& \boldsymbol{u}(0)=\boldsymbol{u}(T), \quad \boldsymbol{d}(0)=\boldsymbol{d}(T) \quad \text { in } \Omega .
\end{aligned}
$$

In order to arrive at the previous variational formulation, the following equalities have been used:

$$
\nabla \cdot(\nabla \boldsymbol{d} \odot \nabla \boldsymbol{d})=\nabla\left(\frac{|\nabla \boldsymbol{d}|^{2}}{2}\right)+\nabla \boldsymbol{d}^{t} \Delta \boldsymbol{d}
$$

and

$$
\left(\nabla\left(\frac{|\nabla \boldsymbol{d}|^{2}}{2}\right), \mathbf{v}\right)=0 \quad \forall \mathbf{v} \in \boldsymbol{V}
$$


Notice that the reproductivity conditions $\boldsymbol{u}(0)=\boldsymbol{u}(T)$ and $\boldsymbol{d}(0)=\boldsymbol{d}(T)$ have sense, because $\boldsymbol{u}$ and $\boldsymbol{d}$ are (at least weakly) continuous functions from $[0, T]$ onto some Banach spaces.

\subsection{Weak Maximum Principle for $d$}

An essential characteristic of the problem for $\boldsymbol{d}$ (given $\boldsymbol{u}$ ) is the following weak maximum principle:

Lemma 2.1 Assume (4). Then, given $\boldsymbol{u} \in L^{2}(0, T ; \boldsymbol{V}) \cap L^{\infty}(0, T ; \boldsymbol{H})$, any weak solution for the $\boldsymbol{d}$-problem, i.e. $\boldsymbol{d} \in L^{2}\left(0, T ; \boldsymbol{H}^{1}\right) \cap L^{\infty}\left(0, T ; \boldsymbol{L}^{2}\right)$ such that

$$
\begin{aligned}
& \left\langle\partial_{t} \boldsymbol{d}, \boldsymbol{e}\right\rangle+((\boldsymbol{u} \cdot \nabla) \boldsymbol{d}, \boldsymbol{e})+(f(\boldsymbol{d}), \boldsymbol{e})+(\nabla \boldsymbol{d}, \nabla \boldsymbol{e})=0, \quad \forall \boldsymbol{e} \in \boldsymbol{H}_{0}^{1}, \\
& \boldsymbol{d}_{\mid \partial \Omega \times(0, T)}=\boldsymbol{h} \quad \text { and } \quad \boldsymbol{d}(0)=\boldsymbol{d}(T),
\end{aligned}
$$

verifies $|\boldsymbol{d}(x, t)| \leq 1$ a.e. in $\Omega \times(0, T)$.

\section{Proof.}

Let us define the function:

$$
\varphi(x, t)=\left(|\boldsymbol{d}(x, t)|^{2}-1\right)_{+}
$$

where $z_{+}=\max (z, 0)$ for each $z \in \mathbb{R}$. Hypothesis $|\mathbf{h}| \leq 1$ on $\partial \Omega \times(0, T)$ implies that also $\varphi=0$ on $\partial \Omega \times(0, T)$. Taking $\mathbf{e}=\varphi \boldsymbol{d}$ as test function (5), one has:

$$
\frac{1}{2} \int_{\Omega} \partial_{t}\left(|\boldsymbol{d}|^{2}\right) \varphi+\int_{\Omega}(\boldsymbol{u} \cdot \nabla)|\boldsymbol{d}|^{2} \varphi+\int_{\Omega} \nabla \boldsymbol{d}: \nabla(\varphi \boldsymbol{d})+\int_{\Omega} \varphi f(\boldsymbol{d}) \cdot \boldsymbol{d}=0
$$

The first three terms of (7) can be written as follows:

$$
\begin{aligned}
\frac{1}{2} \int_{\Omega} \partial_{t}\left(|\boldsymbol{d}|^{2}\right) \varphi & =\frac{1}{2} \int_{\Omega} \partial_{t}\left(|\boldsymbol{d}|^{2}-1\right) \varphi=\frac{1}{4} \frac{d}{d t}\|\varphi\|_{L^{2}(\Omega)}^{2}, \\
\int_{\Omega}(\boldsymbol{u} \cdot \nabla)|\boldsymbol{d}|^{2} \varphi=\int_{\Omega}(\boldsymbol{u} \cdot \nabla)\left(|\boldsymbol{d}|^{2}-1\right) \varphi=\int_{\Omega}(\boldsymbol{u} \cdot \nabla) \varphi \cdot \varphi=0 & \\
\int_{\Omega} \nabla \boldsymbol{d}: \nabla(\varphi \boldsymbol{d}) & =\frac{1}{2} \int_{\Omega} \nabla\left(|\boldsymbol{d}|^{2}\right) \cdot \nabla \varphi+\int_{\Omega}|\nabla \boldsymbol{d}|^{2} \varphi \\
& \geq \frac{1}{2} \int_{\Omega} \nabla\left(|\boldsymbol{d}|^{2}-1\right) \nabla \varphi=\frac{1}{2}\|\nabla \varphi\|_{L^{2}(\Omega)}^{2} .
\end{aligned}
$$

Taking into account in the last term of $(7)$ that $f(\boldsymbol{d}) \cdot \boldsymbol{d}>0=\frac{1}{\varepsilon^{2}}\left(|\boldsymbol{d}|^{2}-1\right)|\boldsymbol{d}|^{2}$ as $|\boldsymbol{d}|>1$ (and $f(\boldsymbol{d}) \cdot \boldsymbol{d}=0$ as $|\boldsymbol{d}| \leq 1$ ), we arrive at the differential inequality:

$$
\frac{d}{d t}\|\varphi\|_{L^{2}}^{2}+2\|\nabla \varphi\|_{L^{2}}^{2} \leq 0
$$


Integrating in $[0, T]$ one has

$$
\|\varphi(T)\|_{L^{2}}^{2}+2 \int_{0}^{T}\|\nabla \varphi\|_{L^{2}}^{2} \leq\|\varphi(0)\|_{L^{2}}^{2}
$$

Since $\varphi(T)=\varphi(0)$, the previous inequality implies $\nabla \varphi=0$ a.e. in $\Omega \times(0, T)$. Therefore $\varphi(\cdot, t) \equiv$ constant in $\Omega$. But, since $\varphi(\cdot, t)=0$ on $\partial \Omega$ (thanks to $|\boldsymbol{d}|=$ $|\mathbf{h}| \leq 1$ on $\partial \Omega$, we conclude $\varphi(x, t)=0$ a.e. in $(x, t) \in \Omega \times(0, T)$, i.e. $|\boldsymbol{d}(x, t)| \leq 1$ a.e. in $\Omega \times(0, T)$.

Notice that, although the constraint $|\boldsymbol{d}| \leq 1$ has been explicitly included in the problem (1)-(3), the previous Lemma say us that it is not necessary because this constraint can be obtained a posteriori.

In the sequel, we will consider the penalized function $f$ as a bounded function, since from maximum principle one has $|\boldsymbol{d}| \leq 1$, hence one can consider the problem (1) changing $f$ by $\tilde{f}$ the auxiliary function

$$
\tilde{f}(\boldsymbol{d})=\left\{\begin{array}{lll}
f(\boldsymbol{d}) & \text { if } & |\boldsymbol{d}| \leq 1 \\
0 & \text { if } & |\boldsymbol{d}|>1
\end{array}\right.
$$

Indeed, if $(\boldsymbol{u}, p, \boldsymbol{d})$ is a solution of (1) with $\tilde{f}$, in particular $|\boldsymbol{d}| \leq 1$ (because the maximum principle is also verified, since $\tilde{f}(\boldsymbol{d}) \cdot \boldsymbol{d}>0$ as $|\boldsymbol{d}|>1)$, then $(\boldsymbol{u}, p, \boldsymbol{d})$ is also a solution of (1) with $f$. The inverse implication is easy to verify .

Notice that, $|\widetilde{f}(\boldsymbol{d})| \leq 1 / \varepsilon^{2}$ for each $\boldsymbol{d} \in \mathbb{R}^{N}$, hence all estimates below will be dependent on $\varepsilon$ (recall that the asymptotic behavior as $\varepsilon \rightarrow 0$ for reproductive solutions remains as open problem).

\subsection{Variational Formulacion in $u$ and $\hat{d}$}

Since a time-dependent boundary data $\boldsymbol{h}(x, t)$ on $\partial \Omega \times(0, T)$ has been considered, an adequate lifting is necessary. Assuming $\boldsymbol{h} \in H^{1}\left(0, T ; \boldsymbol{H}^{1 / 2}(\partial \Omega)^{N}\right)$, if we define $\tilde{\boldsymbol{d}}(t)$ as the weak solution of the Laplace-Dirichlet problem

$$
\begin{cases}-\Delta \widetilde{\boldsymbol{d}}=0 & \text { in } \Omega, \\ \left.\widetilde{\boldsymbol{d}}\right|_{\partial \Omega}=\boldsymbol{h}(t) & \text { on } \partial \Omega,\end{cases}
$$

then $\tilde{\boldsymbol{d}} \in H^{1}\left(0, T ; \boldsymbol{H}^{1}(\Omega)^{N}\right)$ (notice that only weak regularity of this Laplace-Dirichlet problem is used, therefore it is suffices Lipschitz regularity for the domain $\Omega$ ). Moreover, since $\mathbf{h}(0)=\mathbf{h}(T)$ on $\partial \Omega$, then $\tilde{\boldsymbol{d}}(0)=\tilde{\boldsymbol{d}}(T)$ in $\Omega$.

Therefore, if we define $\widehat{\boldsymbol{d}}(t)=\boldsymbol{d}(t)-\tilde{\boldsymbol{d}}(t)$, then $\widehat{\boldsymbol{d}}(t) \in \boldsymbol{H}_{0}^{1}(\Omega)^{N}, \Delta \widehat{\boldsymbol{d}}=\Delta \boldsymbol{d}$ in $\Omega \times(0, T)$ and $\boldsymbol{d}(0)=\boldsymbol{d}(T)$ if and only if $\hat{\boldsymbol{d}}(0)=\hat{\boldsymbol{d}}(T)$. Then, we can rewrite the problem in the variables $(\boldsymbol{u}, \widehat{\boldsymbol{d}})$ as follows: 
Notice that $(\underset{\sim}{\boldsymbol{u}}, \boldsymbol{d})$ is a weak reproductive solution of (1)-(3) if and only if $(\boldsymbol{u}, \widehat{\boldsymbol{d}})$ with $\boldsymbol{d}=\widehat{\boldsymbol{d}}+\tilde{\boldsymbol{d}}(\tilde{\boldsymbol{d}}$ previously defined $)$ verifies

$$
\begin{aligned}
& \boldsymbol{u} \in L^{2}(\boldsymbol{V}) \cap L^{\infty}(\boldsymbol{H}), \\
& \widehat{\boldsymbol{d}} \in L^{\infty}\left(\boldsymbol{H}_{0}^{1}\right), \quad \Delta \widehat{\boldsymbol{d}} \in L^{2}\left(\boldsymbol{L}^{2}\right),
\end{aligned}
$$

and

$$
\begin{aligned}
& \left\langle\partial_{t} \boldsymbol{u}, \mathbf{v}\right\rangle+((\boldsymbol{u} \cdot \nabla) \boldsymbol{u}, \mathbf{v})+(\nabla \boldsymbol{u}, \nabla \mathbf{v})+\left(\nabla \boldsymbol{d}^{t} \Delta \widehat{\boldsymbol{d}}, \mathbf{v}\right)=0 \quad \forall \mathbf{v} \in \boldsymbol{V} \cap \boldsymbol{L}^{\infty}, \\
& \left\langle\partial_{t} \widehat{\boldsymbol{d}}, \mathbf{e}\right\rangle+((\boldsymbol{u} \cdot \nabla) \boldsymbol{d}, \mathbf{e})+(f(\boldsymbol{d}), \mathbf{e})-(\Delta \widehat{\boldsymbol{d}}, \mathbf{e})=-\left(\partial_{t} \widetilde{\boldsymbol{d}}, \mathbf{e}\right) \quad \forall \mathbf{e} \in \boldsymbol{L}^{3}, \\
& \boldsymbol{u}(0)=\boldsymbol{u}(T), \quad \widehat{\boldsymbol{d}}(0)=\widehat{\boldsymbol{d}}(T) \quad \text { in } \Omega
\end{aligned}
$$

\subsection{Energy Inequality}

Taking $\mathbf{v}=\boldsymbol{u}$ and $\mathbf{e}=-\boldsymbol{\Delta} \widehat{\boldsymbol{d}}$ as test functions in previous formulation, one has

$$
\left\{\begin{array}{l}
\left(\partial_{t} \boldsymbol{u}, \boldsymbol{u}\right)+((\boldsymbol{u} \cdot \nabla) \boldsymbol{u}, \boldsymbol{u})+(\nabla \boldsymbol{u}, \nabla \boldsymbol{u})+\left(\nabla \boldsymbol{d}^{t} \Delta \widehat{\boldsymbol{d}}, \boldsymbol{u}\right)=0 \\
-\left(\partial_{t} \widehat{\boldsymbol{d}}, \Delta \widehat{\boldsymbol{d}}\right)-((\boldsymbol{u} \cdot \nabla) \boldsymbol{d}, \Delta \widehat{\boldsymbol{d}})-(f(\boldsymbol{d}), \Delta \widehat{\boldsymbol{d}})+(\Delta \widehat{\boldsymbol{d}}, \Delta \widehat{\boldsymbol{d}})=\left(\partial_{t} \widetilde{\boldsymbol{d}}, \Delta \widehat{\boldsymbol{d}}\right)
\end{array}\right.
$$

Adding up, taking into account that

$$
((\boldsymbol{u} \cdot \nabla) \boldsymbol{u}, \boldsymbol{u})=0 \quad \text { and } \quad\left(\nabla \boldsymbol{d}^{t} \Delta \widehat{\boldsymbol{d}}, \boldsymbol{u}\right)-((\boldsymbol{u} \cdot \nabla) \boldsymbol{d}, \Delta \widehat{\boldsymbol{d}})=0,
$$

one arrives (at least formally) at the following energy equality:

$$
\frac{1}{2} \frac{d}{d t}\left(\|\boldsymbol{u}\|_{L^{2}}^{2}+\|\nabla \widehat{\boldsymbol{d}}\|_{L^{2}}^{2}\right)+\|\nabla \boldsymbol{u}\|_{L^{2}}^{2}+\|\Delta \widehat{\boldsymbol{d}}\|_{L^{2}}^{2}=(f(\boldsymbol{d}), \Delta \widehat{\boldsymbol{d}})+\left(\partial_{t} \tilde{\boldsymbol{d}}, \Delta \widehat{\boldsymbol{d}}\right) .
$$

Consequently, one has the energy inequality:

$$
\frac{d}{d t}\left(\|\boldsymbol{u}\|_{L^{2}}^{2}+\|\nabla \widehat{\boldsymbol{d}}\|_{L^{2}}^{2}\right)+2\|\nabla \boldsymbol{u}\|_{L^{2}}^{2}+\|\Delta \widehat{\boldsymbol{d}}\|_{L^{2}}^{2} \leq 2\left(\|f(\boldsymbol{d})\|_{L^{2}}^{2}+\left\|\partial_{t} \tilde{\boldsymbol{d}}\right\|_{L^{2}}^{2}\right),
$$

where the right hand side is bounded in $L^{1}(0, T)$ since $\partial_{t} \tilde{\boldsymbol{d}} \in L^{2}\left(L^{2}\right)$.

\section{The Main Result}

Theorem 3.1 Let $T>0$ and $\Omega \subset \mathbb{R}^{N}(N=2$ or 3$)$ an open bounded domain with Lipschitz boundary. Assume $\boldsymbol{h} \in H^{1}\left(0, T ; \boldsymbol{H}^{1 / 2}(\partial \Omega)\right)$ such that (4) hold. Then there exists a weak reproductive solution $(\boldsymbol{u}, \boldsymbol{d})$ of problem (1)-(3) 
Remark. Hypothesis of regularity for boundary data $\boldsymbol{h}$ can be relaxed by

$$
\boldsymbol{h} \in L^{\infty}\left(0, T ; \boldsymbol{H}^{1 / 2}(\partial \Omega)\right) \quad \text { with } \quad \partial_{t} \boldsymbol{h} \in L^{2}\left(0, T ; \boldsymbol{L}^{2}(\partial \Omega)\right) .
$$

Indeed, this regularity implies the following regularity for the lifting function

$$
\widetilde{\boldsymbol{d}} \in L^{\infty}\left(0, T ; \boldsymbol{H}^{1}(\Omega)\right) \quad \text { with } \quad \partial_{t} \tilde{\boldsymbol{d}} \in L^{2}\left(0, T ; \boldsymbol{L}^{2}(\Omega)\right),
$$

which will be sufficient in the sequel. Notice that regularity for time derivative can be proved using the "transposition solution" of the Laplace-Dirichlet problem with boundary data equal to $\partial_{t} \mathbf{h}$ (due to $\partial_{t} \mathbf{h}$ has not a trace sense). By the contrary, further regularity for the domain $\Omega$ is necessary (which implies to have $H^{2}$-regularity for the adjoint problem), in order to define the transposition solution.

In the proof of this theorem, the Galerkin method will be used. Firstly, we consider the initial-boundary problem associated to arbitrary initial data. Afterwards, the key is to find certain initial data at $t=0$ that are "reproduced" at final time $t=T$.

\subsection{The Galerkin Initial-Boundary Problem}

Let $\left\{\phi_{i}\right\}_{n} \geq 1$ and $\left\{\varphi_{i}\right\}_{n} \geq 1$ "special" basis of $\boldsymbol{V}$ and $\boldsymbol{H}_{0}^{1}(\Omega)$, respectively, formed by eigenfunctions of the Stokes problem

$$
\left(\nabla \phi_{i}, \nabla \mathbf{v}\right)=\lambda_{i}\left(\phi_{i}, \mathbf{v}\right) \quad \forall \mathbf{v} \in \boldsymbol{V}, \phi_{i} \in \boldsymbol{V}, \quad \text { con }\left\|\phi_{i}\right\|_{L^{2}}=1, \quad \lambda_{i} \nearrow+\infty
$$

and of the Poisson problem

$$
\left(\nabla \varphi_{i}, \nabla \mathbf{w}\right)=\mu_{i}\left(\varphi_{i}, \mathbf{w}\right) \quad \forall \mathbf{w} \in \boldsymbol{H}_{0}^{1}, \varphi_{i} \in \boldsymbol{H}^{1}, \quad \text { con }\left\|\varphi_{i}\right\|_{L^{2}}=1, \quad \mu_{i} \nearrow+\infty
$$

Let $\boldsymbol{V}^{m}$ and $\boldsymbol{W}^{m}$ be the finite-dimensional subspaces spanned by $\left\{\phi_{1}, \phi_{2}, \ldots, \phi_{n}\right\}$ and $\left\{\varphi_{1}, \varphi_{2}, \ldots, \varphi_{n}\right\}$ respectively.

Given $\boldsymbol{u}_{0} \in \boldsymbol{H}$ and $\boldsymbol{d}_{0} \in \boldsymbol{H}^{1}$ (verifying the compatibility condition $\left.\boldsymbol{d}_{0}\right|_{\partial \Omega}=\mathbf{h}(0)$ ), for each $m \geq 1$, we seek an approximate solution $\left(\boldsymbol{u}_{m}, \boldsymbol{d}_{m}\right)$, with $\boldsymbol{u}_{m}:[0, T] \mapsto \boldsymbol{V}^{m}$ and $\boldsymbol{d}_{m}=\widehat{\boldsymbol{d}}_{m}+\widetilde{\boldsymbol{d}}$, with $\widehat{\boldsymbol{d}}_{m}:[0, T] \mapsto \boldsymbol{W}^{m}$, verifying the following variational formulation a.e. in $t$ :

$$
\left\{\begin{array}{c}
\left(\partial_{t} \boldsymbol{u}_{m}(t), \mathbf{v}_{m}\right)+\left(\left(\boldsymbol{u}_{m}(t) \cdot \nabla\right) \boldsymbol{u}_{m}(t), \mathbf{v}_{m}\right)+\left(\nabla \boldsymbol{u}_{m}(t), \nabla \mathbf{v}_{m}\right) \\
+\left(\nabla \boldsymbol{d}_{m}^{t}(t) \Delta \widehat{\boldsymbol{d}}_{m}(t), \mathbf{v}_{m}\right)=0 \quad \forall \mathbf{v}_{m} \in \boldsymbol{V}^{m} \\
\left(\partial_{t} \widehat{\boldsymbol{d}}_{m}(t), \mathbf{e}_{m}\right)+\left(\left(\boldsymbol{u}_{m}(t) \cdot \nabla\right) \boldsymbol{d}_{m}(t), \mathbf{e}_{m}\right)+\left(f\left(\boldsymbol{d}_{m}(t)\right), \mathbf{e}_{m}\right) \\
+\left(\nabla \widehat{\boldsymbol{d}}_{m}(t), \nabla \mathbf{e}_{m}\right)=-\left(\partial_{t} \widetilde{\boldsymbol{d}}(t), \mathbf{e}_{m}\right) \quad \forall \mathbf{e}_{m} \in \boldsymbol{W}^{m} \\
\boldsymbol{u}_{m}(0)=\boldsymbol{u}_{0 m}=P_{m}\left(\boldsymbol{u}_{0}\right), \quad \boldsymbol{d}_{m}(0)=\boldsymbol{d}_{0 m}=Q_{m}\left(\boldsymbol{d}_{0}\right),
\end{array}\right.
$$


Here, $P_{m}: \boldsymbol{H} \mapsto \boldsymbol{V}^{m}$ denotes the usual orthogonal projector of $\boldsymbol{H}$ onto $\boldsymbol{V}^{m}$, and $Q_{m}: \boldsymbol{L}^{2} \mapsto \boldsymbol{W}^{m}$ the orthogonal projector of $\boldsymbol{L}^{2}$ onto $\boldsymbol{W}^{m}$. In particular, $\boldsymbol{u}_{0 m} \rightarrow \boldsymbol{u}_{0}$ in $\boldsymbol{L}^{2}$ and $\boldsymbol{d}_{0 m} \rightarrow \boldsymbol{d}_{0}$ in $\boldsymbol{H}^{1}$ (as $m \rightarrow 0$ ).

If we put

$$
\boldsymbol{u}_{m}(t)=\sum_{j=1}^{m} \xi_{i, m}(t) \phi_{i} \quad \text { and } \quad \widehat{\boldsymbol{d}}_{m}(t)=\sum_{j=1}^{m} \zeta_{i, m}(t) \varphi_{i},
$$

then (9) can be rewritten as a first order ordinary differential system (in normal form) associated to the unknowns $\left(\xi_{i, m}(t), \zeta_{i, m}(t)\right)$. Then, one has existence of a maximal solution (defined in some interval $\left[0, \tau_{m}\right) \subset[0, T]$ ) of the related Cauchy problem. Moreover, from a priori estimates (independent on $m$ ) which will be obtained below, in particular one has that $\tau_{m}=T$. Finally, using regularity of the chosen spectral basis, uniqueness of approximate solution will be proved in section 3.3.

Remark. Since a discretization in space has been done in definition of approximate solution, the maximum principle is not always verified, therefore the constraint $\left|\boldsymbol{d}_{m}\right| \leq 1$ is not true in general.

\section{2 "A priori" estimates}

Taking $\boldsymbol{u}_{m}(t) \in \boldsymbol{V}^{m}$ as test function in the $\boldsymbol{u}$-system of $(9)$ and $-\Delta \widehat{\boldsymbol{d}}_{m}(t) \in \boldsymbol{W}^{m}$ in the $\boldsymbol{d}$-system (latter is possible due to consider the special eigenfunction basis), and following the argument that yields to energy inequality (Section 2.4), one has

$$
\frac{d}{d t}\left(\left\|\boldsymbol{u}_{m}\right\|_{L^{2}}^{2}+\left\|\nabla \widehat{\boldsymbol{d}}_{m}\right\|_{L^{2}}^{2}\right)+2\left\|\nabla \boldsymbol{u}_{m}\right\|_{L^{2}}^{2}+\left\|\Delta \widehat{\boldsymbol{d}}_{m}\right\|_{L^{2}}^{2} \leq 2\left(\left\|f\left(\boldsymbol{d}_{m}\right)\right\|_{L^{2}}^{2}+\left\|\partial_{t}\right\|_{L^{2}}^{2}\right)
$$

hence, using the initial estimates $\left\|\boldsymbol{u}_{m}(0)\right\|_{L^{2}}^{2} \leq C$ and $\left\|\nabla \widehat{\boldsymbol{d}}_{m}(0)\right\|_{L^{2}}^{2} \leq C$, Gronwall's lemma implies

$$
\left(\boldsymbol{u}_{m}\right) \text { is uniformly bounded in } L^{\infty}(\boldsymbol{H}) \cap L^{2}(\boldsymbol{V})
$$

and

$$
\left(\widehat{\boldsymbol{d}}_{m}\right) \text { is uniformly bounded in } L^{\infty}\left(\boldsymbol{H}_{0}^{1}\right) \cap L^{2}\left(\boldsymbol{H}^{2}\right)
$$

Therefore, since $\boldsymbol{d}_{m}=\widehat{\boldsymbol{d}}_{m}+\tilde{\boldsymbol{d}}$ with $\tilde{\boldsymbol{d}} \in L^{\infty}\left(\boldsymbol{H}^{1}\right)$ and $\Delta \boldsymbol{d}_{m}=\Delta \widehat{\boldsymbol{d}}_{m}$, one has

$$
\left(\boldsymbol{d}_{m}\right) \text { is uniformly bounded in } L^{\infty}\left(\boldsymbol{H}^{1}\right)
$$

and

$$
\left(\Delta \boldsymbol{d}_{m}\right) \text { is uniformly bounded in } L^{2}\left(\boldsymbol{L}^{2}\right) .
$$

Using previous estimations in (9), it is easy to obtain the following estimations:

$$
\left(\partial_{t} \boldsymbol{u}_{m}\right) \text { is uniformly bounded in } L^{2}\left(\left(\boldsymbol{V} \cap \boldsymbol{L}^{\infty}\right)^{\prime}\right)
$$


and

$$
\left(\partial_{t} \widehat{\boldsymbol{d}}_{m}\right) \text { is uniformly bounded in } L^{2}\left(\boldsymbol{L}^{3 / 2}\right)
$$

Using compactness results for time spaces with values in Banach spaces (see [12]) with the triplet $\boldsymbol{V} \hookrightarrow \boldsymbol{H} \hookrightarrow\left(\boldsymbol{V} \cap \boldsymbol{L}^{\infty}\right)^{\prime}$ and $\boldsymbol{H}^{2} \cap \boldsymbol{H}_{0}^{1} \hookrightarrow \boldsymbol{H}_{0}^{1} \hookrightarrow \boldsymbol{L}^{3 / 2}$, one has

$$
\left(\boldsymbol{u}_{m}\right) \text { is relatively compact in } L^{2}(\boldsymbol{H})
$$

and

$$
\left(\widehat{\boldsymbol{d}}_{m}\right) \text { is relatively compact in } L^{2}\left(\boldsymbol{H}_{0}^{1}\right) \text {. }
$$

Consequently, $\left(\boldsymbol{d}_{m}\right)$ is relatively compact in $L^{2}\left(\boldsymbol{H}^{1}\right)$.

In fact, this compactness is sufficient in the pass to the limit in (9) in order to control the nonlinear terms.

Remark. Notice that if $\boldsymbol{h}$ and $\Omega$ are regular enough, then $\tilde{\boldsymbol{d}} \in L^{2}\left(\boldsymbol{H}^{2}\right)$ and $\left(\boldsymbol{d}_{m}\right)$ is bounded in $L^{2}\left(\boldsymbol{H}^{2}\right)$.

\subsection{Uniqueness of Approximate Solution}

Without less of generality, in this section only the $3 D$ case $(N=3)$ will be considered. Let $\left(\boldsymbol{u}_{m}^{1}, \boldsymbol{d}_{m}^{1}\right)$ and $\left(\boldsymbol{u}_{m}^{2}, \boldsymbol{d}_{m}^{2}\right)$ be two solutions of (9), and we denote $\boldsymbol{u}_{m}=$ $\boldsymbol{u}_{m}^{1}-\boldsymbol{u}_{m}^{2}$ and $\boldsymbol{d}_{m}=\boldsymbol{d}_{m}^{1}-\boldsymbol{d}_{m}^{2}$ (notice that $\boldsymbol{d}_{m}=\widehat{\boldsymbol{d}}_{m}$ ). Making the difference between (9) for $\left(\boldsymbol{u}_{m}^{1}, \boldsymbol{d}_{m}^{1}\right)$ and $\left(\boldsymbol{u}_{m}^{2}, \boldsymbol{d}_{m}^{2}\right)$, considering $\boldsymbol{u}_{m}$ and $-\Delta \boldsymbol{d}_{m}$ as test functions, and taking into account the equalities

$$
\begin{aligned}
\left(\left(\boldsymbol{u}_{m}^{1} \cdot \nabla\right) \boldsymbol{u}_{m}^{1}-\left(\boldsymbol{u}_{m}^{2} \cdot \nabla\right) \boldsymbol{u}_{m}^{2}, \boldsymbol{u}_{m}\right) & =\left(\left(\boldsymbol{u}_{m}^{1} \cdot \nabla\right) \boldsymbol{u}_{m}+\left(\boldsymbol{u}_{m} \cdot \nabla\right) \boldsymbol{u}_{m}^{2}, \boldsymbol{u}_{m}\right) \\
& =\left(\left(\boldsymbol{u}_{m} \cdot \nabla\right) \boldsymbol{u}_{m}^{2}, \boldsymbol{u}_{m}\right),
\end{aligned}
$$

and

$$
\begin{aligned}
& \left(\Delta \boldsymbol{d}_{m}^{1} \cdot \nabla \boldsymbol{d}_{m}^{1}-\Delta \boldsymbol{d}_{m}^{2} \cdot \nabla \boldsymbol{d}_{m}^{2}, \boldsymbol{u}_{m}\right)+\left(\left(\boldsymbol{u}_{m}^{1} \cdot \nabla\right) \boldsymbol{d}_{m}^{1}-\left(\boldsymbol{u}_{m}^{2} \cdot \nabla\right) \boldsymbol{d}_{m}^{2},-\Delta \boldsymbol{d}_{m}\right) \\
= & \left(\Delta \boldsymbol{d}_{m}^{1} \cdot \nabla \boldsymbol{d}_{m}+\Delta \boldsymbol{d}_{m} \cdot \nabla \boldsymbol{d}_{m}^{2}, \boldsymbol{u}_{m}\right)+\left(\left(\boldsymbol{u}_{m}^{1} \cdot \nabla\right) \boldsymbol{d}_{m}+\left(\boldsymbol{u}_{m} \cdot \nabla\right) \boldsymbol{d}_{m}^{2},-\Delta \boldsymbol{d}_{m}\right) \\
= & \left(\Delta \boldsymbol{d}_{m}^{1} \cdot \nabla \boldsymbol{d}_{m}, \boldsymbol{u}_{m}\right)+\left(\left(\boldsymbol{u}_{m}^{1} \cdot \nabla\right) \boldsymbol{d}_{m},-\Delta \boldsymbol{d}_{m}\right),
\end{aligned}
$$

the following equality holds

$$
\begin{aligned}
& \frac{1}{2} \frac{d}{d t}\left(\left\|\boldsymbol{u}_{m}\right\|_{L^{2}}^{2}+\left\|\nabla \boldsymbol{d}_{m}\right\|_{L^{2}}^{2}\right)+\left\|\nabla \boldsymbol{u}_{m}\right\|_{L^{2}}^{2}+\left\|\Delta \boldsymbol{d}_{m}\right\|_{L^{2}}^{2} \\
& =-\left(\left(\boldsymbol{u}_{m} \cdot \nabla\right) \boldsymbol{u}_{m}^{2}, \boldsymbol{u}_{m}\right)-\left(\left(\Delta \boldsymbol{d}_{m}^{1} \cdot \nabla\right) \boldsymbol{d}_{m}, \boldsymbol{u}_{m}\right) \\
& +\left(\left(\boldsymbol{u}_{m}^{1} \cdot \nabla\right) \boldsymbol{d}_{m}, \Delta \boldsymbol{d}_{m}\right)-\left(f\left(\boldsymbol{d}_{m}^{1}\right)-f\left(\boldsymbol{d}_{m}^{2}\right), \Delta \boldsymbol{d}_{m}\right) .
\end{aligned}
$$


We bound each term on the right hand side of (11) as follows:

$$
\begin{aligned}
\left|\left(\left(\boldsymbol{u}_{m} \cdot \nabla\right) \boldsymbol{u}_{m}, \boldsymbol{u}_{m}^{2}\right)\right| & \leq\left\|\boldsymbol{u}_{m}\right\|_{L^{4}} \cdot\left\|\nabla \boldsymbol{u}_{m}\right\|_{L^{2}} \cdot\left\|\boldsymbol{u}_{m}^{2}\right\|_{L^{4}} \\
& \leq\left\|\boldsymbol{u}_{m}\right\|_{L^{2}}^{1 / 4} \cdot\left\|\boldsymbol{u}_{m}\right\|_{H^{1}}^{7 / 4} \cdot\left\|\boldsymbol{u}_{m}^{2}\right\|_{L^{4}} \\
& \leq \bar{\varepsilon}\left\|\boldsymbol{u}_{m}\right\|_{H^{1}}^{2}+C_{\bar{\varepsilon}}\left\|\boldsymbol{u}_{m}^{2}\right\|_{L^{4}}^{8} \cdot\left\|\boldsymbol{u}_{m}\right\|_{L^{2}}^{2} . \\
\left|\left(\left(\Delta \boldsymbol{d}_{m}^{1} \cdot \nabla\right) \boldsymbol{d}_{m}, \boldsymbol{u}_{m}\right)\right| & \leq\left\|\Delta \boldsymbol{d}_{m}^{1}\right\|_{L^{3}} \cdot\left\|\nabla \boldsymbol{d}_{m}\right\|_{L^{6}} \cdot\left\|\boldsymbol{u}_{m}\right\|_{L^{2}} \\
& \leq C\left\|\Delta \boldsymbol{d}_{m}^{1}\right\|_{L^{3}} \cdot\left\|\nabla \boldsymbol{d}_{m}\right\|_{H^{1}} \cdot\left\|\boldsymbol{u}_{m}\right\|_{L^{2}} \\
& \leq \bar{\varepsilon}\left\|\Delta \boldsymbol{d}_{m}\right\|_{L^{2}}^{2}+C_{\bar{\varepsilon}}\left\|\Delta \boldsymbol{d}_{m}^{1}\right\|_{L^{3}}^{2} \cdot\left\|\boldsymbol{u}_{m}\right\|_{L^{2}}^{2} . \\
\left|\left(\left(\boldsymbol{u}_{m}^{1} \cdot \nabla\right) \boldsymbol{d}_{m}, \Delta \boldsymbol{d}_{m}\right)\right| & \leq\left\|\boldsymbol{u}_{m}^{1}\right\|_{L^{4}} \cdot\left\|\nabla \boldsymbol{d}_{m}\right\|_{L^{4}} \cdot\left\|\Delta \boldsymbol{d}_{m}\right\|_{L^{2}} \\
& \leq C\left\|\boldsymbol{u}_{m}^{1}\right\|_{L^{4}} \cdot\left\|\Delta \boldsymbol{d}_{m}\right\|_{L^{2}}^{7 / 4} \cdot\left\|\nabla \boldsymbol{d}_{m}\right\|_{L^{4}}^{1 / 4} \\
& \leq \bar{\varepsilon}\left\|\Delta \boldsymbol{d}_{m}\right\|_{L^{2}}^{2}+C_{\bar{\varepsilon}}\left\|\boldsymbol{u}_{m}^{1}\right\|_{L^{4}}^{8} \cdot\left\|\nabla \boldsymbol{d}_{m}\right\|_{L^{2}}^{2} .
\end{aligned}
$$

In order to bound $\left(f\left(\boldsymbol{d}_{m}^{1}\right)-f\left(\boldsymbol{d}_{m}^{2}\right), \Delta \boldsymbol{d}_{m}\right)$ we will use the following equality:

$$
\begin{aligned}
f\left(\boldsymbol{d}_{m}^{1}\right)-f\left(\boldsymbol{d}_{m}^{2}\right) & =\frac{1}{\varepsilon^{2}}\left(\left|\boldsymbol{d}_{m}^{1}\right|^{2} \boldsymbol{d}_{m}^{1}-\left|\boldsymbol{d}_{m}^{2}\right|^{2} \boldsymbol{d}_{m}^{2}-\boldsymbol{d}_{m}\right) \\
\left( \pm\left|\boldsymbol{d}_{m}^{1}\right|^{2} \boldsymbol{d}_{m}^{2}\right) & =\frac{1}{\varepsilon^{2}} g\left(\boldsymbol{d}_{m}^{1}, \boldsymbol{d}_{m}^{2}\right) \boldsymbol{d}_{m}
\end{aligned}
$$

where $g\left(\boldsymbol{d}_{m}^{1}, \boldsymbol{d}_{m}^{2}\right)=\left|\boldsymbol{d}_{m}^{1}\right|^{2}+\left(\boldsymbol{d}_{m}^{1}+\boldsymbol{d}_{m}^{2}\right) \boldsymbol{d}_{m}^{2}-1$. In particular, $\left|g\left(\boldsymbol{d}_{m}^{1}, \boldsymbol{d}_{m}^{2}\right)\right| \leq 4$. Therefore

$$
\begin{aligned}
\left|\left(f\left(\boldsymbol{d}_{m}^{1}\right)-f\left(\boldsymbol{d}_{m}^{2}\right), \Delta \boldsymbol{d}_{m}\right)\right| & \leq\left|\left(\frac{1}{\varepsilon^{2}} g\left(\boldsymbol{d}_{m}^{1}, \boldsymbol{d}_{m}^{2}\right) \boldsymbol{d}_{m}, \Delta \boldsymbol{d}_{m}\right)\right| \\
& \leq \frac{4}{\varepsilon^{2}}\left\|\boldsymbol{d}_{m}\right\|_{L^{2}}\left\|\Delta \boldsymbol{d}_{m}\right\|_{L^{2}} \\
& \leq \bar{\varepsilon}\left\|\Delta \boldsymbol{d}_{m}\right\|_{L^{2}}^{2}+\frac{C(\bar{\varepsilon})}{\varepsilon^{4}}\left\|\nabla \boldsymbol{d}_{m}\right\|_{L^{2}}^{2}
\end{aligned}
$$

Accordingly (13)-(15) and choosing $\bar{\varepsilon}$ small enough, one has

$$
\left\{\begin{array}{l}
\frac{d}{d t}\left(\left\|\boldsymbol{u}_{m}\right\|_{L^{2}}^{2}+\left\|\nabla \boldsymbol{d}_{m}\right\|_{L^{2}}^{2}\right) \leq a_{m}(t)\left(\left\|\boldsymbol{u}_{m}\right\|_{L^{2}}^{2}+\left\|\nabla \boldsymbol{d}_{m}\right\|_{L^{2}}^{2}\right) \\
\left\|\boldsymbol{u}_{m}(0)\right\|_{L^{2}}^{2}+\left\|\nabla \boldsymbol{d}_{m}(0)\right\|_{L^{2}}^{2}=0 .
\end{array}\right.
$$

with $a_{m}(t)$ bounded in $\in L^{1}(0, T)$ (using that $\left\|\Delta \boldsymbol{d}_{m}^{1}\right\|_{L^{3}}^{2},\left\|\boldsymbol{u}_{m}^{2}\right\|_{L^{4}}^{8}$ and $\left\|\boldsymbol{u}_{m}^{1}\right\|_{L^{4}}^{8}$ are bounded in $\left.L^{1}(0, T)\right)$. Applying Gronwall's Lemma, one has $\boldsymbol{u}_{m}=0$ and $\nabla \boldsymbol{d}_{m}=0$. Finally, since $\boldsymbol{d}_{m}=0$ on $\partial \Omega$, then $\boldsymbol{d}_{m}=0$. Therefore, uniqueness of approximate solution for the initial-boundary problem is proved. 


\subsection{Existence of approximate reproductive solution.}

From energy inequality (10), one has in particular

$$
\frac{d}{d t}\left(\left\|\boldsymbol{u}_{m}\right\|_{L^{2}}^{2}+\left\|\nabla \widehat{\boldsymbol{d}}_{m}\right\|_{L^{2}}^{2}\right)+C\left(\left\|\boldsymbol{u}_{m}\right\|_{L^{2}}^{2}+\left\|\nabla \widehat{\boldsymbol{d}}_{m}\right\|_{L^{2}}^{2}\right) \leq 2\left(\left\|f\left(\boldsymbol{d}_{m}\right)\right\|_{L^{2}}^{2}+\left\|\partial_{t} \tilde{\boldsymbol{d}}\right\|_{L^{2}}^{2}\right) .
$$

Multiplying by $e^{C t}$ and integrating in $[0, T]$ :

$$
\begin{aligned}
e^{C T}\left(\left\|\boldsymbol{u}_{m}(T)\right\|_{L^{2}}^{2}+\left\|\nabla \hat{\boldsymbol{d}}_{m}(T)\right\|_{L^{2}}^{2}\right) & \leq\left\|\boldsymbol{u}_{m}(0)\right\|_{L^{2}}^{2}+\left\|\nabla \hat{\boldsymbol{d}}_{m}(0)\right\|_{L^{2}}^{2} \\
& +2 \int_{0}^{T} e^{C t}\left(\left\|f\left(\boldsymbol{d}_{m}\right)\right\|_{L^{2}}^{2}+\left\|\partial_{t} \widetilde{\boldsymbol{d}}\right\|_{L^{2}}^{2}\right) .
\end{aligned}
$$

Given $\left(\boldsymbol{u}_{0}^{m}, \boldsymbol{d}_{0}^{m}\right) \in V^{m} \times W^{m}$, we define the map

$$
\begin{aligned}
L^{m}:[0, T] & \mapsto \mathbb{R}^{m} \times \mathbb{R}^{m} \\
t & \mapsto\left(\xi_{1 m}(t), \ldots, \xi_{m m}(t), \zeta_{1 m}(t), \ldots, \zeta_{m m}(t)\right)
\end{aligned}
$$

where $\left(\xi_{1 m}(t), \ldots, \xi_{m m}(t)\right)$ and $\left(\zeta_{1 m}(t), \ldots, \zeta_{m m}(t)\right)$ are coefficients of $\boldsymbol{u}_{m}(t)$ and $\widehat{\boldsymbol{d}}_{m}(t)$ respect to $\boldsymbol{V}^{m}$ and $\boldsymbol{W}^{m}$ respectively, being $\left(\boldsymbol{u}_{m}(t), \widehat{\boldsymbol{d}}_{m}(t)\right)$ the (unique) approximate solution of (9) corresponding to the initial data $\left(\boldsymbol{u}_{0}^{m}, \boldsymbol{d}_{0}^{m}\right)$.

Now, varying the initial data $\left(\boldsymbol{u}_{0}^{m}, \boldsymbol{d}_{0}^{m}\right)$, we are going to define a new map $\Phi^{m}$ : $\mathbb{R}^{m} \times \mathbb{R}^{m} \mapsto \mathbb{R}^{m} \times \mathbb{R}^{m}$ as follows: given $L_{0}^{m} \in \mathbb{R}^{m} \times \mathbb{R}^{m}$, we define $\Phi^{m}\left(L_{0}^{m}\right)=L^{m}(T)$, where $L^{m}(t)$ is related to the solution of problem (9) with initial data $L_{0}^{m}\left(=L^{m}(0)\right)$.

By uniqueness of approximate solution of the initial-boundary problem, this map is well-defined. Moreover, using regularity of the corresponding ordinary differential system (equivalent to (9)), this map is continuous.

In order to prove existence of fixed point of $\Phi^{m}$, we will use Leray-Schauder's Theorem. Consequently, we have to prove that for all $\lambda \in[0,1]$, solutions $L_{0}^{m}(\lambda)$ of

$$
L_{0}^{m}(\lambda)=\lambda \Phi^{m}\left(L_{0}^{m}(\lambda)\right)
$$

are uniformly bounded (independent of $\lambda$ ). Since $L_{0}^{m}(0)=\{0\}$, it is suffices to analyse $\lambda \in(0,1]$ and the equation

$$
\frac{1}{\lambda} L_{0}^{m}(\lambda)=\Phi^{m}\left(L_{0}^{m}(\lambda)\right)
$$

Considering the norm $\left\|L^{m}(t)\right\|_{\mathbf{R}^{m} \times \mathbf{R}^{m}}=\left(\left\|\boldsymbol{u}_{m}(t)\right\|_{L^{2}}^{2}+\left\|\nabla \widehat{\boldsymbol{d}}_{m}(t)\right\|_{L^{2}}^{2}\right)^{1 / 2}$ in $\mathbb{R}^{m} \times \mathbb{R}^{m}$, inequality (16) yields

$$
e^{C T}\left\|\frac{1}{\lambda} L_{0}^{m}(\lambda)\right\|_{\mathbf{R}^{m} \times \mathbf{R}^{m}}^{2} \leq\left\|L_{0}^{m}(\lambda)\right\|_{\mathbf{R}^{m} \times \mathbf{R}^{m}}^{2}+K(T),
$$


where $K(T)$ is an upper bound of $2 \int_{0}^{T} e^{C t}\left(\left\|f\left(\boldsymbol{d}_{m}\right)\right\|_{L^{2}}^{2}+\left\|\partial_{t} \tilde{\boldsymbol{d}}\right\|_{L^{2}}^{2}\right) d t$.

Since $\lambda \in(0,1]$, one has

$$
\left\|L_{0}^{m}(\lambda)\right\|_{\mathbf{R}^{m} \times \mathbf{R}^{m}}^{2} \leq \frac{K(T)}{e^{C T}-1}
$$

which is a bound independent of $\lambda$ (and $m$ ). Consequently, Leray-Schauder Theorem implies the existence of fixed point of $\Phi^{m}$, that is existence of reproductive Galerkin solution.

Moreover, for each approximate reproductive solution $\left(\boldsymbol{u}_{m}, \boldsymbol{d}_{m}\right)$, their corresponding initial-end data is bounded in the $L^{2} \times H^{1}$-norm, i.e $\left\|\left(\boldsymbol{u}_{m}, \widehat{\boldsymbol{d}}_{m}\right)(0)\right\|_{L^{2} \times H^{1}} \leq$ $C$ (independent of $m$ ). Therefore, the estimations obtained in Section 3.2 hold for the approximate reproductive solutions.

\subsection{Pass to the limit in reproductive approximate solutions}

The pass to the limit in variational formulation (9) can be done as in [8], using estimations and compactness obtained in Section 3.2 (independents of $m$ ) in order to control nonlinear terms. Consequently, here we will only write the pass to the limit in reproductive conditions.

¿From estimations of $\left(\boldsymbol{d}_{m}\right)$ in $L^{\infty}\left(\boldsymbol{H}^{1}\right)$ and $\left(\partial_{t} \boldsymbol{d}_{m}\right)$ in $L^{2}\left(\boldsymbol{L}^{3 / 2}\right)$ and using the triplet of spaces $\boldsymbol{H}^{2} \hookrightarrow \boldsymbol{L}^{2} \hookrightarrow \boldsymbol{L}^{3 / 2}$, one has ([12]) that $\left(\boldsymbol{d}_{m}\right)$ is relatively compact in $C\left([0, T] ; \boldsymbol{L}^{2}\right)$, hence $\boldsymbol{d}_{m}(T) \rightarrow \boldsymbol{d}(T)$ and $\boldsymbol{d}_{m}(0) \rightarrow \boldsymbol{d}(0)$ in $\boldsymbol{L}^{2}(\Omega)$. Since $\boldsymbol{d}_{m}(T)=$ $\boldsymbol{d}_{m}(0)$, then $\boldsymbol{d}(T)=\boldsymbol{d}(0)$ in $\boldsymbol{L}^{2}(\Omega)$. Moreover, it is easy to see that $\boldsymbol{d} \in C_{w}\left([0, T] ; \boldsymbol{H}^{1}\right)$ (i.e. $\boldsymbol{d}$ is continuous from $[0, T]$ onto $\boldsymbol{H}^{1}$, respect to the weak topology in $\boldsymbol{H}^{1}$ ), therefore $\boldsymbol{d}(T)=\boldsymbol{d}(0)$ in $\boldsymbol{H}^{1}(\Omega)$. The argument for $\boldsymbol{u}$ is similar.

Consequently, we have found a weak reproductive solution of problem (1) - (3).

\section{Relation between reproductive and periodic so- lutions}

\subsection{The $2 D$ case}

When the domain $\Omega \subset \mathbb{R}^{2}$, uniqueness of weak solution for the initial-boundary problem (associated to any initial data $\boldsymbol{u}_{0}$ and $\boldsymbol{d}_{0}$ ) can be showed.

Consequently, given a reproductive solution $(\boldsymbol{u}, \boldsymbol{d})$ associated to $\boldsymbol{u}(0)=\boldsymbol{u}(T):=$ $\boldsymbol{u}_{0}$ and $\boldsymbol{d}(0)=\boldsymbol{d}(T):=\boldsymbol{d}_{0}$, then $(\boldsymbol{u}, \boldsymbol{d})$ is the (unique) solution of the initial-boundary problem associated to the initial data $\left(\boldsymbol{u}_{0}, \boldsymbol{d}_{0}\right)$, which is defined in all time $t \in(0, \infty)$. Therefore, this solution is T-periodic, because for instance in $(T, 2 T)$ must be equal 
to the reproductive solution such that $\boldsymbol{u}(T)=\boldsymbol{u}(2 T)=\boldsymbol{u}_{0}$ and $\boldsymbol{d}(T)=\boldsymbol{d}(2 T)=\boldsymbol{d}_{0}$, etc.

Finally, using regularity of solution $(\boldsymbol{u}, \boldsymbol{d})$ for strictly positive times (see [4] for the Navier-Stokes equations and [8] for the nematic liquid crystal model), it is easy to prove that every periodic solution is regular, assuming regularity on boundary data $\boldsymbol{h}$.

\subsection{The $3 D$ case}

For the Navier-Stokes problem (i.e. $\boldsymbol{d}=0$ ), it is known an argument that provides (global in time) regularity of any reproductive solution associated to regular and small enough data [6]. In [11] this argument is applied to an abstract parabolic problem, which is a generalization of some of the main hydrodynamic models.

The argument is based in three main properties of the model:

1. Integrating in $(0, T)$ the weak energy inequality, the terms $\|\boldsymbol{u}(T)\|_{L^{2}}^{2}-\|\boldsymbol{u}(0)\|_{L^{2}}^{2}$ vanish thanks to the reproductivity, hence an estimate is deduced for the $L^{2}\left(0, T ; \boldsymbol{H}^{1}(\Omega)\right)$-norm of velocity $\boldsymbol{u}$, which is decreasing to zero as data goes to zero, but for any reproductive initial value.

2. From previous estimate, and using an mean value argument, one has that there exists $t_{\star} \in(0, T)$ such that $\left\|\boldsymbol{u}\left(t_{\star}\right)\right\|_{H^{1}} \leq \bar{\varepsilon}$ small enough.

3. Using the global regularity for the initial value problem with small and regular data, the strong solution associated to the model with initial data $\boldsymbol{u}\left(t_{\star}\right)$ is defined in $\left[t_{\star},+\infty\right)$ and remains small in all times $t \geq t_{\star}$. Moreover, this solution coincides with the weak reproductive solution $\boldsymbol{u}$ in $\left[t_{\star}, T\right]$, thanks to the weak-strong uniqueness result of the model. Therefore $\boldsymbol{u}(T)($ and $\boldsymbol{u}(0)$ ) is small respect to the $H^{1}$-norm. Consequently $\boldsymbol{u}$ is regular and periodic in the whole $[0,+\infty)$.

But now, for the liquid crystal model (1)-(3) in $\Omega \subset \mathbb{R}^{3}$, it is not clear how regularity of reproductive solution can be proved, because previous argument (point 1 ) is not valid. The problem is that smallness of the right hand-side of energy inequality (8) cannot be assured, because of the term $\|f(\boldsymbol{d})\|_{L^{2}}^{2}$ (which is bounded by not small). Indeed, integrating in $(0, T)$ and using the reproductivity (now the different constants of problem are considered), one arrives at

$$
\lambda \gamma \int_{0}^{T}\|\Delta \widehat{\boldsymbol{d}}\|_{L^{2}}^{2} \leq \int_{0}^{T} C\left(\lambda \gamma\|f(\boldsymbol{d})\|_{L^{2}}^{2}+\left\|\partial_{t} \tilde{\boldsymbol{d}}\right\|_{L^{2}}^{2}\right) \leq \bar{\varepsilon}+C \lambda \gamma \int_{0}^{T}\|f(\boldsymbol{d})\|_{L^{2}}^{2}
$$

where $\bar{\varepsilon}$ is small enough. But this bound is not small, even taking $\mu$ or $\gamma$ big enough. Only, previous right hand-side is small when penalty parameter $\varepsilon$ is big enough, which is not a physical interesting case. 
On the other hand, we can consider other energy inequality (see [8] for the case of time-independent boundary data $\boldsymbol{h}$ ):

$$
\begin{aligned}
& \frac{d}{d t}\left(\|\boldsymbol{u}\|_{L^{2}}^{2}+\lambda\|\nabla \widehat{\boldsymbol{d}}\|_{L^{2}}^{2}+2 \lambda \int_{\Omega} F(d)\right)+2 \mu\|\nabla \boldsymbol{u}\|_{L^{2}}^{2} \\
& +\lambda \gamma\|\Delta \widehat{\boldsymbol{d}}-f(\boldsymbol{d})\|_{L^{2}}^{2} \leq \frac{\lambda}{\gamma} \int_{0}^{T}\left\|\partial_{t} \tilde{\boldsymbol{d}}\right\|_{L^{2}}^{2}+\frac{2 \lambda}{\varepsilon^{2}} \int_{0}^{T}\left\|\partial_{t} \tilde{\boldsymbol{d}}\right\|_{L^{1}}
\end{aligned}
$$

which can be obtained considering $\boldsymbol{u}$ as test function in the $\boldsymbol{u}$-system of problem and $\lambda(-\Delta \widehat{\boldsymbol{d}}+f(\boldsymbol{d}))$ in the $\boldsymbol{d}$-system, where $F(\boldsymbol{d})=\left(|\boldsymbol{d}|^{2}-1\right)^{2} / 4 \varepsilon^{2}$ and $f(\boldsymbol{d})=\nabla_{\boldsymbol{d}} F(\boldsymbol{d})$ is used. Indeed, one has

$\frac{d}{d t}\left(\|\boldsymbol{u}\|_{L^{2}}^{2}+\lambda\|\nabla \widehat{\boldsymbol{d}}\|_{L^{2}}^{2}\right)+2 \lambda \int_{\Omega} \partial_{t} \widehat{\boldsymbol{d}} \cdot f(\boldsymbol{d})+2 \mu\|\nabla \boldsymbol{u}\|_{L^{2}}^{2}+\lambda \gamma\|\Delta \widehat{\boldsymbol{d}}-f(\boldsymbol{d})\|_{L^{2}}^{2} \leq \frac{\lambda}{\gamma}\left\|\partial_{t} \tilde{\boldsymbol{d}}\right\|_{L^{2}}^{2}$

Then,

$$
\int_{\Omega} \partial_{t} \widehat{\boldsymbol{d}} \cdot f(\boldsymbol{d})=\frac{d}{d t} \int_{\Omega} F(\boldsymbol{d})+\int_{\Omega} \partial_{t} \tilde{\boldsymbol{d}} \cdot f(\boldsymbol{d})
$$

Therefore, bounded the last term by $\frac{1}{\varepsilon^{2}}\left\|\partial_{t} \tilde{\boldsymbol{d}}\right\|_{L^{1}}$, inequality (17) is proven.

In this case, smallness for $\int_{0}^{T}\|\Delta \widehat{\boldsymbol{d}}-f(\boldsymbol{d})\|_{L^{2}}^{2}$ can be obtained (if $\int_{0}^{T}\left\|\partial_{t} \tilde{\boldsymbol{d}}\right\|_{L^{2}}^{2}$ and $\int_{0}^{T}\left\|\partial_{t} \tilde{\boldsymbol{d}}\right\|_{L^{1}}$ are small), but this does not give sufficient information in order to obtain smallness of the $H^{2}$ norm of $\hat{\boldsymbol{d}}$ (again the term $\|f(\boldsymbol{d})\|_{L^{2}}^{2}$ appears).

In conclusion, existence of periodic solution (and regularity) remains as an open problem.

Acknowledgments: The authors has been partially supported by D.G.E.S. and M.C. y T. (Spain), Projet BFM2003-06446. Moreover, third author is also supported by the projet 301354/03-0 CNPq. (Brasil).

\section{References}

[1] F. Bethuel, H. Brezis, F. Hélein, Asymptotics for the minimization of a Ginzburg-Landau functional, Calc. Var. 1 (1993), 123-148.

[2] D. Coutand, S. Shkoller, Well posedness of the full Ericsen-Leslye model of nematic liquid crystals, Note C.R.A.S, t. 333, Serie I (2001), 919-924.

[3] F. Guillén-González, M.A. Rojas-Medar, Global solution of nematic liquid crystal models, Note C.R.A.S, t. 335, Serie I (2002), 1-6.

[4] J. G. Heywood, The Navier-Stokes equations: on the existence regularity and decay of solutions, Ind. Univer. Math. Journal, 25(5) (1980), 639-681. 
[5] S. Kaniel, M. Shinbrot, A reproductive property of Navier-Stokes equations, Arch. Rational Mech. Anal., 24 (1967), 362-369.

[6] H. Kato Existence of periodic solutions of the Navier-Stokes equations, J. Math. Anal. Appl. 208 (1997), 141-157.

[7] F.H. Lin Nonlinear theory of defects in nematic liquid crystals: phase transition and flow phenomena, Comm. Pure Appl. Math., 42 (1989), 789-814.

[8] F.H. Lin, C. Liu Non-parabolic dissipative systems modelling the flow of liquid crystals, Comm. Pure Appl. Math., 4 (1995), 501-537.

[9] F.H. Lin, C. Liu Existence of solutions for the Ericksen-Leslie system, Arch. Rat. Mech. Anal., 154 (2000), 135-156.

[10] J.L. Lions Quelques Méthods de Résolution des Problémes aux limits non linéares, Dunod, Paris, 1969.

[11] G. Lukaszewicz, E. E. Ortega-Torres, M. A. Rojas-Medar, Strong periodic solutions for a class of abstract evolution equations, Nonlinear Anal. 54, 6 (2003), 1045-1056.

[12] J. Simon Compact sets in the space $L^{p}(0, T ; B)$, Annali di Matematica Pure ed Applicata (IV), Vol. CXLVI (1987), 65-96. 